\title{
Study of Propagation of Light along an Acupuncture Meridian
}

\author{
Chunho Choi* and Kwang-Sup Soh \\ School of Physics, Seoul National University, \\ Seoul 151-747, KOREA \\ Sang Min Lee \\ Department of Biomedical Engineering, Hanyang University, \\ Seoul 133-791, KOREA \\ Gilwon Yoon \\ Department of Electronics $\&$ Information, Seoul National University of Technology, \\ Seoul 139-743, KOREA
}

(Received September 24, 2003)

\begin{abstract}
Propagation of light along a pericardium meridian is measured and compared with that along a non-meridian path one $\mathrm{cm}$ away from the meridian. Source-light is illuminated on the NeiGuan(PC6) and propagated light is detected at three points along the meridian. Four reference points are one $\mathrm{cm}$ away from the corresponding meridian points, respectively. Light propagates better along the meridian than the reference path with more than twenty percent difference for all the subjects tested.
\end{abstract}

OCIS codes : $170.3660,170.4580,170.5280$.

\section{INTRODUCTION}

The acupuncture points and meridian systems are fundamental in diagnosis and treatment in traditional Chinese medicine. Their physiological functions are nowadays widely accepted, but their histological existence is not established $[1,2]$, and their mechanism is mostly undetermined [3-5]. Nevertheless there have been many efforts since the 1950 s to investigate their electrical properties by measuring impedances [6], which clearly show that meridians and acupuncture points have higher electric conductivity [7]. There are also other kinds of scientific investigations such as higher carbon dioxide emission rate at the acupuncture points [8], radio isotope trace flows [9], and relations with functional MRI images [10]. Notwithstanding with all these efforts, in order to firmly establish the physical existence of and to uncover the mechanism of the acupuncture points and meridians, we need more experiments for investigating their various physical and physiological aspects.

Recently, the possibility of optical means was reported $[11,12]$, where diffuse reflection coefficient of acupuncture points is shown to be different from non-acupuncture points, i.e., light propagates better along the meridians. There are also indication of biophotons spontaneously coming out from the human skin $[13,14]$. Thus the optical characteristics of acupuncture points and meridians are worthwhile more investigation.

In this paper we report the better propagation of light along the pericardium meridian compared with nearby reference skin. We detected and compared light intensities which diffused along the two lines. Our result is consistent with Kaznachiev's results, and invites further finer investigations.

\section{METHOD AND PROCEDURE}

The experimental setup is shown in Fig. 1. All experiments were performed in a dark room. The light source is a Tungsten-Halogen lamp (TS-428, Acton Research Co.) which produces a non-coherent white light, and was modulated in $10 \mathrm{~Hz}$ by an optical chopper (SR540, Stanford Research Systems).

The modulated light was delivered to a point (S) on the skin of an arm through an optical fiber (SPEX 


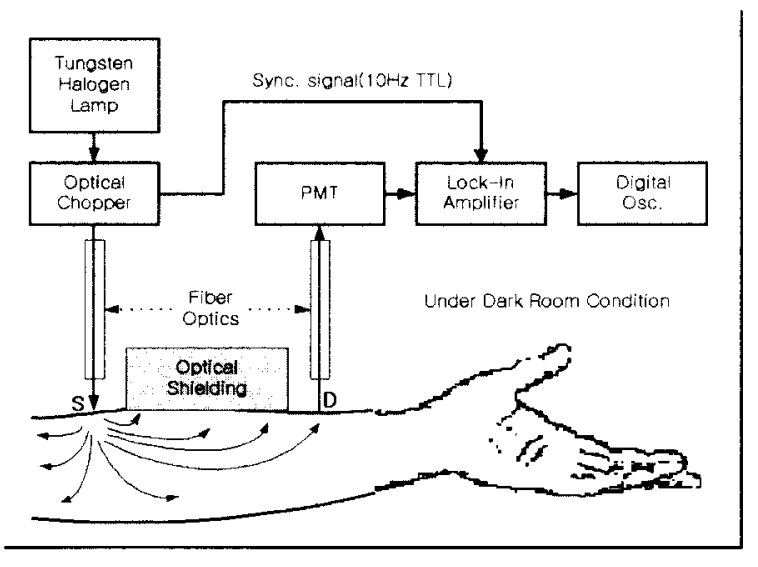

FIG. 1. Experimental Setup. The modulated light from Tungsten-Halogen lamp was delivered to the points (S) by an optical fiber. Photo multiplier tube is used as a detector. Signals from PMT were discriminated and amplified by lock-in amplifier, and recorded on the digital storage oscilloscope. SPEX fiber with $1 \mathrm{~mm}$ core diameter was used as source fiber, and $3 \mathrm{~mm}$ multiple core fiber was used to enlarge the detection area. The skin between source and detector fiber was covered with light absorbing material.

fiber) of $1 \mathrm{~mm}$ core diameter. The light is then assumed to propagate (or diffuse) inside the skin, especially well along the meridian. We detect the light at another point (D) on the skin using a multiple core optical fiber of $3 \mathrm{~mm}$ diameter to enlarge the detection area. The skin between the source point (S) and the detection point (D) is covered with light absorbing cloths for optical shielding.

The detecting fiber is connected to a photo multiplier tube (PMT) with spectral range of 165-850 nm (C962 Channel Photomultiplier, Perkin-Elmer Optoelectronics). The signals from the PMT are discriminated and amplified by a lock-in amplifier (SR850, Stanford Research Systems) which is locked to the optical chopper for $10 \mathrm{~Hz}$ TTL. The signals are recorded on the digital storage oscilloscope.

Pericardium meridian runs along the center of the forearm. We locate according to the traditional acupuncture chart. Detection point (D) is the NeiGuan (PC6), and the reference point $\mathrm{O}$, one $\mathrm{cm}$ off from the pericardium meridian line. There are three source points (S) along the meridian (PC5, a non acupuncture point, and PC4), and the corresponding three reference points are chosen one $\mathrm{cm}$ away from the three meridian points. These six source points are illuminated by the tungsten-halogen lamp light through the source-optical fiber. The distance between the nearest two source points along the meridian is one cun, which is a measuring unit traditionally used in acupuncture treatment. One cun is the length between the middle phalangeal flexure and the distal phalangeal flexure of the middle finger. The distance

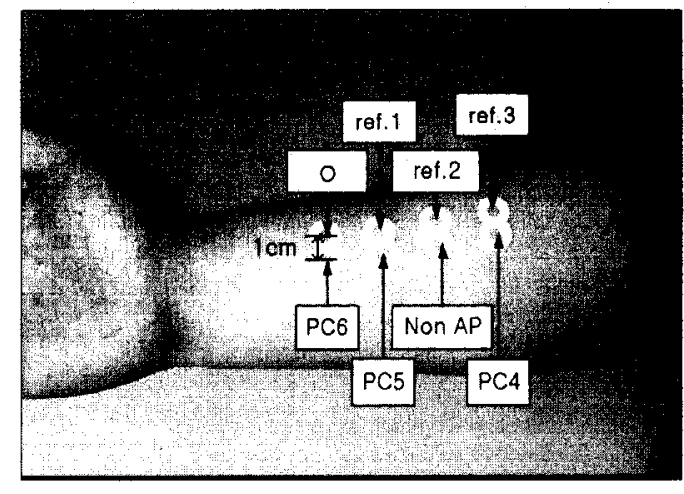

FIG. 2. Measurement Points along the Pericardium Meridian and Non-meridian reference line. Detector fiber is located on PC6 and $\mathrm{O}$, and other points along each line were irradiated by the source fiber, respectively.

from elbow flexure to wrist flexure is twelve "cun". The Nei-Guan (PC6) is located on the forearm, two "cun" away from the center of the wrist. Jian-Shi (PC5) and Xi-Men (PC4) are the acupuncture points of the pericardium meridian.

Each subject was kept balanced in the dark room for ten minutes with labels on the measurement points ( $S$ and $D$ ) along the pericardium meridian and the reference line. The same measurements were repeated three times for each subject. Between the measurements the subject was kept balanced in the dark room for two minutes. The room temperature was about $23^{\circ} \mathrm{C}$.

\section{RESULTS}

Fourteen healthy subjects (age 25-47; 10 males and 4 females) were tested as volunteers. The data were shown in the Table 1. The currents are measured in $\mathrm{mV}$ unit, and its fluctuation was on the average $0.03 \pm 0.03 \mathrm{mV}$. The measured currents at the three meridian points ( $\mathrm{PC} 5, \mathrm{D} 2, \mathrm{PC} 4)$, and their respective reference points (ref. point 1, ref. point 2, ref. point 3 ) are listed in the Table, and they are compared by the $\%$ difference, $\left(\left(V_{\text {meridian }}-V_{\text {ref }}\right) / V_{\text {meridian }}\right) \times 100$. In the first case of PC5 and the reference point 1 , the propagation along the meridian was about $20 \%$ more than the propagation along the reference line. In the second case of the non-acupuncture D2 on the pericardium meridian, and the reference point 2 , we found surprisingly $69 \%$ stronger propagation. This remarkable phenomena was consistent for all the fourteen subjects. At the third points of $\mathrm{PC} 4$ and the reference point 3 , the intensity was also stronger at the meridian points, but the detected currents are close to the noise level of the system.

In the Fig. 3 we show the average and the standard deviation of the measured currents in units of $\mathrm{mV}$ for 
TABLE 1. Comparison between the meridian and the reference points. The points PC5, D2, PC4 are on the pericardium meridian, and the reference points $1,2,3$ are $1 \mathrm{~cm}$ off from the meridian points, respectively.

\begin{tabular}{l|cccccc|ccc}
\hline \hline & \multicolumn{7}{c}{ PMT output $(\mathrm{mV})$} & \multicolumn{3}{c}{ \%ratios $=\frac{\left(V_{M}-V_{R}\right)}{V_{M}} \times 100$} \\
\hline & PC5 & ref.1 & D2 & ref.2 & PC4 & ref.3 & PC5/ref.1 & D2/ref.2 & PC4/ref.3 \\
\hline Sub.1 & 69.01 & 49.12 & 4.91 & 0.59 & 0.20 & 0.21 & 28.8 & 87.9 \\
\hline Sub.2 & 58.43 & 50.02 & 3.12 & 0.89 & 0.16 & 0.32 & 14.4 & 71.4 & -101 \\
\hline Sub.3 & 55.06 & 35.74 & 2.55 & 0.46 & 0.62 & 0.37 & 35.1 & 81.8 & 40.3 \\
\hline Sub.4 & 57.15 & 49.62 & 1.05 & 0.74 & 0.69 & 0.35 & 13.2 & 28.9 & 48.5 \\
\hline Sub.5 & 59.25 & 66.62 & 16.35 & 7.85 & 0.59 & 0.38 & -12.4 & 52.0 & 35.7 \\
\hline Sub.6 & 67.47 & 62.69 & 16.08 & 3.98 & 0.66 & 0.28 & 7.1 & 75.2 & 56.9 \\
\hline Sub.7 & 71.31 & 39.72 & 6.67 & 0.90 & 0.21 & 0.20 & 44.3 & 86.5 & 2.1 \\
\hline Sub.8 & 56.56 & 31.10 & 3.17 & 0.50 & 0.19 & 0.13 & 45.0 & 84.1 & 31.2 \\
\hline Sub.9 & 74.08 & 44.41 & 28.79 & 3.21 & 3.07 & 0.26 & 40.0 & 88.8 & 91.5 \\
\hline Sub.10 & 74.29 & 60.36 & 34.80 & 5.96 & 2.59 & 0.29 & 18.7 & 82.9 & 88.7 \\
\hline Sub.11 & 62.91 & 61.22 & 7.64 & 5.43 & 0.40 & 0.25 & 2.7 & 28.9 & 37.6 \\
\hline Sub.12 & 76.74 & 76.07 & 20.05 & 14.56 & 1.08 & 0.85 & 0.9 & 27.4 & 21.1 \\
\hline Sub.13 & 70.80 & 55.08 & 17.15 & 3.04 & 0.74 & 0.30 & 22.2 & 82.3 & 59.7 \\
\hline Sub.14 & 63.73 & 48.51 & 7.58 & 1.15 & 0.35 & 0.21 & 26.2 & 84.9 & 41.0 \\
\hline Avg & $65.5 \pm 7.4$ & $52.2 \pm 12.4$ & $12.1 \pm 10.4$ & $3.5 \pm 3.9$ & $0.8 \pm 0.9$ & $0.3 \pm 0.2$ & $20.4 \pm 17.4$ & $68.8 \pm 23.8$ & $32.0 \pm 47.0$ \\
\hline \hline
\end{tabular}

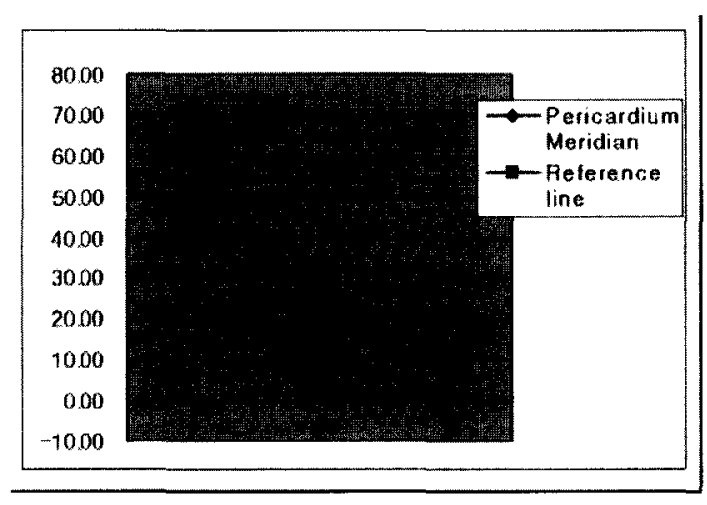

FIG. 3. Experimental Results. Average of Results over 14 Subjects. Numbers on $\mathrm{x}$-axis $(1,2,3)$ denote the source points: PC5, D2, PC4 along pericardium meridian, and ref. 1, ref. 2, ref.3 along non-meridian line, respectively. The unit on $\mathrm{y}$-axis is $\mathrm{mV}$.

the three meridian and three reference points. It clearly indicates that the meridian propagates more light than the reference line.

\section{DISCUSSION}

Our experimental results agree with the Kaznachiev's [11] observation, in the sense that the light propagation along the pericardium meridian is stronger than the nearby reference line. However, in order to confirm the Kaznachiev's claim, further experiments to reveal the light propagation mechanism are needed. For example, one has to study the spectral dependence of light propagation, light intensity dependence, and so on, which we plan to pursue in near future.

For the interpretation and the mechanism of better light propagation along the pericardium meridian, one should consider histological structure and the optical properties of the relevant tissues and interstitial fluids. The pericardium meridian is located between two muscles of a forearm, but at the moment there is not enough knowledge of the optical properties of these physiological structures [15]. Further basic investigations on the light propagation of the relevant biological materials are required. Nevertheless our experiments illustrate that the optical study can be a useful tool for scientific objective investigation of acupuncture and the meridian.

\section{ACKNOWLEDGEMENTS}

The work was supported by grant No. R01-200100023 from the Basic Research Program of the Korea Science \& Engineering Foundation and by grant ITEP A19-02-03 from the Korea Ministry of Commerce, Industry and Energy.

*Corresponding author: gambit@kmc.snu.ac.kr.

\section{REFERENCES}

[1] H. Heine, "Anatomical structure of acupoints," $J$. Trad. Chin. Med., vol. 8, pp. 207-212, 1988

[2] M. Ciszek, J. Szopinski and V. Skrzypulec, "Investigation of morphological structure of acupuncture points 
and meridians," J. Trad. Chin. Med, vol. 5, pp. 289292,1985

[3] C. Shang, "Singular point, organizing center and acupuncture point," Am. J. Chin. Med., vol. 17, pp. $119-127,1989$

[4] J. Fan, S. Xi, Z. Liu and Z. Wei, "The role of gap junctions in determining skin conductance and their possible relationship to acupuncture points and meridians," Am. J. Acupuncture, vol. 18, pp.163-170, 1990

[5] M. - W. Ho and D. P. Knight, "The acupuncture system and the liquid crystalline collagen fibers of the connective tissues," Am. J. Chin. Med., vol. 26, pp. 251-263, 1998

[6] M. Reichmanis, et. al., "Electrical correlates of acupuncture points," IEEE Trans. Biomed. Eng. $B M E$, vol. 22 , pp. 533,1975

[7] W. Zhang, R. Xu and Z. Zhu, "The influence of acupuncture on the impedance measured by four electrodes on meridians," Acup. And Electro-Therapeutics Res. Int. J., vol. 24, pp. 181-188, 1999

[8] W. Zhang, " $\mathrm{CO}_{2}$ emission from skin of meridians," Beijing Biomedical Eng. (in Chinese), vol. 15 pp. 221, 1996

[9] J. -C. Darras, P. de Vernejoul and P. Albareide. "Nuclear Medicine and Acupuncture: a study on the mi- gration of radioactive tracers after injection at acupoints," Am. J. Acupuncture, vol. 20 pp. 245-256, 1992

[10] Z. H. Cho, et. al. "New findings of the correlation between acupoints and corresponding brain cortices using functional MRI," Proc. Natl. Acad. Sci. USA, vol. 95 pp. $2670-2673,1998$

[11] S. Kaznachiev and L. Mochanova, Light from living life and human, (Novosibirsk, Russia, 1998), pp. 101105

[12] H. Lazoura, M. Cohen, E. Lazoura and I. Cosic, "Do Acupuncture Points Have Different Absorption Properties to Laser Light than Surrounding Skin?," in Proc. 2nd IEEE Int. Conf. Bioelectromagnetism, IEEE, Melbourne, Australia, 1998, pp. 172-173

[13] M. Markert, "Whole body measurement of biophoton emission, Biophotonics and Coherent Systems," in Proceedings of the 2nd Additional Contributions, Moscow Univ. Press, 2000, pp. 405-410

[14] C. Choi, et. al. "Biophoton Emission from the Hands," J. of Kor. Phys. Soc. vol. 41(2), pp.275-278, 2002.8

[15] K. -H. Kwon, et al. "The analysis of light coupling and propagation for a composite fiber-dielectric slab with a conductor cladding," J. Opt. Soc. Korea, vol. 7 , no. 2 , pp. $20-27,2003$ 